\title{
Prevalencia de trastornos neurocognitivos en una región rural Argentina
}

\author{
Prevalence of neurocognitive disorders in a rural area of Argentina
}

Prevalência de distúrbios neurocognitivos em uma região rural da Argentina

\author{
Matías Jonás García1,3,4,5, Ana Comesaña ${ }^{2,3,6}$.
}

La cantidad de personas mayores de 60 años crece cada vez más en el mundo. Con el aumento de los mayores, también aparecen más problemáticas de memoria y demencias. En este trabajo buscamos evaluar adultos mayores de una zona de la provincia de Tucumán para detectar adultos con estas problemáticas. Encontramos que hay muchas personas mayores con pérdida de funciones que no han sido diagnosticadas. Hicimos esta investigación puerta a puerta y trabajando con los centros públicos de salud.

\section{Conceptos clave:}

\section{¿Qué se sabe sobre el tema?}

- La proporción de adultos mayores se ha incrementado considerablemente en los últimos años y las previsiones afirman que seguirá creciendo.

- Las enfermedades neurodegenerativas tienen un costo alto para el estado, que aumenta conforme crece la población de adultos mayores con un trastorno neurocognitivo.

- Una evaluación de cribado permite obtener información valiosa sobre el estado mental del paciente y facilita el diagnóstico oportuno.

\section{¿Qué aporta este trabajo?}

- No se han realizado trabajos epidemiológicos sobre trastornos neurocognitivos en la provincia de Tucumán hasta el momento, este estudio es el primero.

-Es un estudio realizado puerta a puerta, evaluando a los pacientes en su ámbito, reduciendo variables estresoras de un ambiente extraño como lo es muchas veces el consultorio.

- Se encontró una prevalencia de trastornos neurocognitivos del $51,17 \%$, superando ampliamente la media nacional, estimada en $12 \%$.

- Este estudio sienta las bases para trabajos epidemiológicos a gran escala mediante el sistema público de salud.

1. Universidad Nacional de Tucumán (UNT). Facultad de Psicología UNT. Tucumán, Argentina

2. Universidad Nacional de Mar del Plata (UNMDP). Facultad de Ciencias de la Salud y Servicio Social UNMDP. Mar del Plata, Argentina.

3. Instituto de Psicología Básica y Aplicada (IPSIBAT) - Centro de investigaciones científicas y tecnológicas (CONICET). Mar del Plata, Argentina.

4. E-mail de contacto: matiasionasg@gmail.com

5. ORCID: https://orcid.org/0000-0002-5276-3208

6. ORCID: https://orcid.org/0000-0002-7505-8851

\section{Resumen:}

Objetivos: realizar un screening cognitivo para detectar casos de trastornos neurocognitivos (TNC) en adultos mayores de 60 años y estimar su prevalencia en el Este de Tucumán. Métodos: Se realizó una evaluación neuropsicológica de cribado. Para el análisis de los datos se conformaron grupos según la presencia y severidad de TNC. Resultados: 257 sujetos participaron en el estudio ( $X=69.9$ años; $D S=7.2 ; 60,7 \%$ género femenino). Se estimó la prevalencia de trastornos neurocognitivos en $51,17 \%$ con un $90 \%$ confianza y una precisión (d) del $4 \%$. Conclusión principal: La prevalencia superó los datos estimados para Argentina. A partir de las pruebas se observó que a menor nivel educativo y a mayor edad, aumentaría el riesgo para desarrollar trastornos neurocognitivos. Este trabajo demostró la capacidad de desarrollar estudios epidemiológicos a gran escala en el sistema sanitario público, articulando la actividad de los diferentes centros de salud, ante la escasez de estudios de este tipo en nuestro país.

Palabras clave: prevalencia; Argentina; anciano; demencia; pruebas de estado mental y demencia.

\section{Abstract:}

Aim: To carry out a cognitive screening to detect cases of Neurocognitive Disorders (NCD) 60 and over years adults and estimate its prevalence in the eastern area of Tucumán. Methods: A neuropsychological assessment was carried out. Groups were formed according to the presence and severity of NCD. Results: 257 subjects participated in the study $(X=69.9$ years, $S D=7.2 ; 60.7 \%$ of female gender $)$. The prevalence of neurocognitive disorders was estimated at $51.17 \%$ with $90 \%$ confidence and a precision (d) of $4 \%$. Main Conclusion: The prevalence exceeded the estimation for Argentina. From the tests it was observed that the lower the educational level and the older the age, the risk of developing neurocognitive disorders would increase. This work demonstrated the ability to develop large-scale epidemiological studies in public health, articulating the activity of the different healthcare centers, given the lack of studies about the matter in our country.

Keywords: prevalence; Argentina; aged; dementia; mental status and dementia tests.

\section{Resumo:}

Objetivos: realizar uma triagem cognitiva para detectar casos de distúrbios neurocognitivos (CTN) em adultos com mais de 60 anos de idade e estimar sua prevalência no leste de Tucumán. Métodos: Foi realizada uma avaliação de triagem neuropsicológica. Para a análise dos dados, os grupos foram formados de acordo com a presença e gravidade da TNC. Resultados: participaram do estudo 257 sujeitos ( $X=$ 69,9 anos; $\mathrm{DP}=7,2 ; 60,7 \%$ do sexo feminino). A prevalência de distúrbios neurocognitivos foi estimada em $51,17 \%$ com $90 \%$ de confiança e precisão (d) de $4 \%$. Conclusão principal: A prevalência excedeu os dados estimados para a Argentina. A partir dos testes, observou-se que quanto menor o nível de escolaridade e maior a idade, aumentaria o risco de desenvolver distúrbios neurocognitivos. Este trabalho demonstrou à capacidade de desenvolver estudos epidemiológicos em larga escala no sistema público de saúde, articulando a atuação dos diferentes centros de saúde, dada a escassez de estudos desse tipo em nosso país.

Palavras-chave: prevalência; Argentina; idoso; demência; testes de estado mental e demencia. 


\section{INTRODUCCIÓN}

La reducción de la mortalidad como así también el incremento en la expectativa de vida por el avance en ciencias de la salud están creando una verdadera revolución demográfica. En el año 2019, las personas mayores de 60 años representaban un $13,46 \%$ de la población mundial(1), proporción que aumenta con una tasa del $3,26 \%$ por año ${ }^{(2)}$. Si bien el crecimiento no ha sido homogéneo en todo el mundo, es claro el avance a nivel global del proceso conocido como envejecimiento demográfico.

De acuerdo con el Informe Mundial sobre el Envejecimiento y la Salud de la Organización Mundial de la Salud(3), las estimaciones indican que entre los años 2000 y 2050, la proporción de población de esa edad pasará del $11 \%$ al $22 \%$ del total. En la Argentina, se estima que los adultos mayores de 60 años conforman un 15,7\% de la población total para este año en curso, el 2020. El aumento en términos absolutos y proporcionales de personas mayores tiene un fuerte impacto en la Salud Pública y la economía de los países, ya que conlleva el incremento de la prevalencia de patologías crónicas no transmisibles relacionadas con la edad. La Organización Panamericana de la Salud(4), señaló la importancia de desarrollar enfoques holísticos y políticas públicas que superen la mirada sobre la enfermedad, la discapacidad y la dependencia para esta franja de la población. Muchas de condiciones médicas dependientes de la edad, como las enfermedades neurodegenerativas (trastornos neurocognitivos o TNC según el manual estadístico de los trastornos mentales DSM-5) $)^{(5)}$, no necesariamente son agudas 0 mortales, pero presentan un fuerte impacto en la calidad de vida de la población a la vez que implican altos costos para la salud pública.

Por ello y en las últimas décadas, el estudio del comportamiento epidemiológico de los TNC se ha convertido en un problema importante y relevante para la salud pública, económica y social ${ }^{(6,7)}$. Conocer sobre la prevalencia, incidencia y tendencias de los TNC en un país, provee información crucial para el desarrollo y planificación de políticas públicas así como permite ahorrar costos en los servicios de salud(6).

En la Argentina, se ha estimado el costo anual de los TNC en US\$ 3.421,4 por cada paciente con diagnóstico de TNC menor y, en US\$ 9.657,6 para los que tienen un TNC mayor. Si se toma en cuenta los casos en los que debe producirse la institucionalización de esa persona, se estima que el costo anual es de US\$ 14.447,68 frente a US\$ 3.189,20 para las personas que no necesitan institucionalización. Los gastos que ocasionarían este tipo de trastornos, se calculan que ocuparían aproximadamente entre el 2,5 al $3 \%$ del PBI del país, para el año $2050^{(8)}$. Asimismo, son escasos los estudios poblacionales sobre la incidencia y prevalencia de los TNC, que se han llevado a cabo en este país. En el año 2011, se publicó el "Primer Registro centralizado de Patologías Cognitivas en Argentina ReDeCar"(9) en donde se registraron los resultados de un estudio piloto que fue realizado con el objetivo de hacer un relevamiento de los pacientes que estaban siendo seguidos en distintas instituciones de salud del país, y que tenían un diagnóstico de algún tipo de TNC. En dicho estudio, los trastornos neurocognitivos menores ocuparon un $17,4 \%$ mientras que los mayores, el $82,6 \%$, del total de los relevados. Aranda y Calabria ${ }^{(8)}$ plantearon que la prevalencia de trastornos neurocognitivos en Argentina está estimada en $12,18 \%$, pero no existen en el país trabajos epidemiológicos completos con toda la información necesaria para la planificación de estrategias en materia de salud pública.

En la provincia de Tucumán, se estimaba en el año 2018 que la población total ascendía a 1.654 .388 , de los cuáles los adultos mayores de 60 años eran 221.838 (el 13,4\%). En el área Este de Tucumán, donde se llevó a cabo este trabajo, la población en ese mismo año era de 321.326 personas y los adultos mayores estaban en el orden de los 37.933 (17,1\%). En esta zona ni en la provincia en general, se cuenta con datos fehacientes sobre la prevalencia de
TNC en adultos mayores; tampoco existen servicios interdisciplinarios en ámbito público ni privado que hagan diagnósticos tempranos o realicen algún seguimiento de las personas que ya cuentan con un diagnóstico de TNC. Los diagnósticos son llevados a cabo, en general, por un profesional médico, que la mayoría de las veces no está especializado ni cuenta con la formación adecuada, para realizar un diagnóstico precoz o detectar los primeros indicadores o signos de los TNC.

EI DSM-5 ${ }^{(5)}$ señala a la evaluación neuropsicológica (ENP) como un elemento diagnóstico fundamental en la detección temprana y precisa, de las patologías de tipo neurodegenerativas ${ }^{(10)}$. A partir de la quinta edición del manual, comienza a utilizarse la denominación "trastornos neurocognitivos" para el grupo de entidades diagnósticas que involucran principalmente la disminución en una o más esferas de la cognición con respecto a un estado anterior de esa persona. Tradicionalmente estos cuadros eran denominados "Deterioro cognitivo leve y demencia", según cómo y cuánto tenga afectada la persona su desempeño en las funciones cognitivas, como así también, la posibilidad de llevar adelante una vida independiente, es decir valerse o no por sí mismo. Entonces, de acuerdo con la gravedad del trastorno, que puede quedar evidenciada a partir de la comparación de su rendimiento en pruebas neuropsicológicas con un grupo poblacional con características semejantes, y la pérdida de la independencia en las actividades de la vida diaria (AVD), estos trastornos pueden clasificarse en menores y mayores. La etiología de los TNC (Enfermedad de Alzheimer, Parkinson, demencia vascular, etc.) ya sean menores o mayores, pasa a ser independiente en la clasificación del DSM-5, evaluándose primero la afección cognitiva y en la vida diaria, luego la etiología del cuadro.

El comportamiento epidemiológico de los TNC varía entre diferentes países y, aún dentro de éstos, se observa variabilidad de una región a otra. Por ello, a la hora de comparar las tasas de prevalencia, deben tenerse en cuenta dos factores ${ }^{(6)}$. En primer lugar, la discrepancia entre las tasas puede reflejar una diferencia en cuanto a los factores de riesgo o de protección que pueden variar en función de la región y las características particulares de un lugar. Sin embargo, también puede reflejar problemas metodológicos y/o diagnósticos. Sobre este último punto recae la importancia de seleccionar técnicas de ENP, teniendo en cuenta su sensibilidad, especificidad y adaptación al contexto socio-cultural en el que se está trabajando, siendo una de las condiciones fundamentales para la validez de los resultados obtenidos ${ }^{(11)}$.

Sobre los factores asociados con una mayor probabilidad de desarrollar un TNC, se han mencionado como factores de riesgo: la edad, un bajo nivel de educación, ocupación laboral previa(12), tabaquismo, obesidad, diabetes mellitus y ser mujer ${ }^{(7)}$; aunque hay autores que disienten sobre la asociación entre el género y los $\mathrm{TNC}^{(13)}$. Por otro lado, algunos estudios han planteado que un alto nivel educativo sería un factor protector ante el deterioro de la cognición ${ }^{(14-16)}$, ya que las experiencias educativas se constituyen como vivencias reforzadoras de la neuroplasticidad cerebral ${ }^{(7,15)}$. La zona de residencia (urbana o rural) y la influencia que tiene este factor en el desarrollo de los TNC, sigue siendo un tema controversial. Algunos autores ${ }^{(15-17)}$, han considerado que la vida en un entorno urbano podría aumentar el riesgo, mientras que estudios más actuales, sitúan al entorno rural como más riesgoso que el urbano ${ }^{(18)}$, planteando que las diferencias en el nivel económico y los estilos de vida entre las dos áreas, podrían justificar esta última idea.

Por todo lo anterior, se podría decir que conocer el comportamiento epidemiológico y realizar un diagnóstico precoz de los TNC, se erige como una necesidad en la población mayor de 60 años, tanto para mejorar su calidad de vida y retrasar la aparición de dichas enfermedades no transmisibles, como para preservar los recursos económicos y asistenciales de los países ${ }^{(8)}$. Por ello, este trabajo tuvo como objetivo realizar un screening cognitivo para detectar casos de TNC en adultos mayores de 60 años y así poder estimar 
su prevalencia en el área Este de la Provincia de Tucumán, durante el año 2018.

\section{MÉTODO}

\section{Diseño y tipo de estudio}

Este estudio fue observacional de corte transversal, para realizar una estimación de la prevalencia de TNC. Los datos fueron recabados desde mediados del 2018 a principios del 2019. Se tomó como unidad de análisis a las personas mayores de 60 años de edad que viviesen en el área Este de la Provincia de Tucumán. Se consideró como criterios de inclusión: edad ( $>60$ años); alfabetización (tener adquirida la lectoescritura); comprensión de órdenes simples; firma del consentimiento informado $\mathrm{y} / \mathrm{o}$ asentimiento para ingresar al estudio. Se excluyeron aquellos sujetos con presencia de trastornos psiquiátricos o neurológicos diagnosticados no asociados con un TNC, así como a los sujetos no alfabetizados.

\section{Selección de población y muestra}

La población de estudio fue dividida en tres bloques, uno por cada localidad, que conforman el Área Este de la provincia de Tucumán. En cada bloque, mediante muestreo aleatorio simple se sortearon los efectores de salud, seleccionando de manera aleatoria manzanas. En cada una de ellas se buscó a los residentes mayores de 60 años hasta completar el número de la muestra estimada.

En virtud de estudios previos realizados ${ }^{(19)}$ se estimó una prevalencia esperada del $12 \%$ para personas mayores de 60 años. Se consignaron 309 sujetos a los que efectivamente se entrevistó y luego de efectuar las correcciones de los protocolos y de excluir los sujetos que no cumplían con los criterios necesarios, la muestra final quedó conformada por 257 casos. Acorde con los datos obtenidos se estimó la prevalencia en esta área con un $90 \%$ de confianza y una precisión del $4 \%$.

\section{Cuestiones éticas}

Este estudio fue desarrollado siguiendo los estándares éticos y de comportamiento de la APA ${ }^{(20)}$, sujeto a la revisión de la Declaración de Helsinki ${ }^{(21)}$ y fue aprobado por el comité metodológico y ético del Sistema Provincial de Salud de Tucumán, donde se realizó el estudio (expediente 1755-615-D-2017).

Se confeccionó un consentimiento informado para el participante o acompañante y un asentimiento para el paciente. Los mismos fueron aprobados junto con el plan de trabajo, por el comité de ética y de investigación ya mencionado.

\section{Materiales}

Se trabajó con datos obtenidos de fuentes primarias, mediante la realización de una entrevista individual de aproximadamente unos 40 a 50 minutos a cada participante en su domicilio. Se tomaron como modelo los instrumentos utilizados en el estudio ReDeCar ${ }^{(9)}$ y también se agregaron algunos más para ampliar la ENP y abarcar otros procesos cognitivos. La entrevista estuvo dividida en dos partes:

\section{Entrevista breve clínica}

La primera parte se utilizó para consignar datos personales (edad; sexo; años de educación; estado civil; con quién vivía la persona, ocupación), y también para recabar los antecedentes clínicos relevantes (hipertensión arterial; consumo de alcohol; parkinsonismo; estados distiroideos; dislipemias; accidente cerebrovascular; traumatismo de cráneo con pérdida de conciencia; enfermedad cardiovascular; presencia de familiar con parentesco de primer grado con diagnóstico de demencia).

\section{Screening cognitivo}

Se realizó una ENP compuesta por las siguientes pruebas o test: Minimental State Examination (MMSE). De acuerdo con las normas de administración consensuadas para Argentina y a los datos normativos establecidos por Butman y colaboradores ${ }^{(22)}$. Este test es uno de los más tradicionales y evalúa la orientación (autopsíquica, en tiempo y lugar), memoria a corto y largo plazo (fijación y recuerdo diferido), atención, lenguaje (comprensión verbal y escrita, expresión verbal -repetición y articulación- y expresión escrita), praxias (por comando escrito y verbal) y habilidades visoconstructivas.

Test del Reloj (23): Evalúa principalmente viso-construcción, atención, habilidad motriz, comprensión y conocimiento de números. Se utilizó la versión de Cacho y García ${ }^{(23)}$, que establece ciertos criterios para la puntuación.

Tareas de fluidez verbal semántica y fonológica: Se utilizaron las tareas de tipo semántico y fonológico por su validez, confiabilidad, sensibilidad y especificidad en estudios locales ${ }^{(24)}$. Se usaron las normas Argentinas de Labos, Trojanowski, del Rio, Zabala y Renato ${ }^{(24)}$.

Trail Making A (test del trazo): Evalúa atención, coordinación visomotora, búsqueda visual, y es sensible a cualquier tipo de daño cerebral. Se utilizaron las normas Argentinas de Fernández, Marino y Alderete $^{(25)}$ según edad y nivel educativo.

\section{RESULTADOS}

Para el análisis de los datos, se conformó en primera instancia una base de datos utilizando Microsoft Excel versión 2010 y el análisis se llevó a cabo utilizando el programa SPSS versión $23.0^{(26)}$. Luego se dividió a los resultados de los participantes en tres grupos, según los puntajes obtenidos en el MMSE siguiendo las normas Argentina de Butman y colaboradores ${ }^{(22)}$, para las cuales un puntaje de entre 27 y 30 es equivalente a normal o sin deterioro; entre 25-26 implica un posible deterioro cognitivo; y entre 10 y 24 puntos una demencia leve a moderada. Entonces, para este estudio los grupos fueron etiquetados de la siguiente manera: el denominado "normal" (27 a 30 puntos) fue definido como "Sin TNC"; el grupo "deterioro leve" (25-26 puntos) es equivalente a "TNC menor"; y el grupo "demencia leve" como "TNC mayor"

La muestra se compuso en un $60,7 \%$ de personas de género femenino. La edad media fue de 69,9 años $(n=257$; $D S=7,28)$, con una escolaridad media de cinco años $(n=257 ; D S=3,03)$.

La prevalencia de TNC para el área Este de la Provincia de Tucumán fue del $51,17 \%(n=256)$ con un $90 \%$ confianza y una precisión (d) del $4 \%$ aproximadamente. Si se divide este porcentaje en TNC menores y mayores, los TNC menores ocuparon un $17,2 \%$ $(n=44)$, siendo menos frecuentes que los mayores, con un $34 \%$ $(\mathrm{n}=87)$.

A continuación se realizaron una serie de análisis para establecer la asociación entre la prevalencia de TNC con género, edad y años de escolaridad. Así, tomando el género como variable de análisis, un análisis porcentual, mostró en hombres una proporción de TNC menor a la hallada en mujeres (fig. 1).

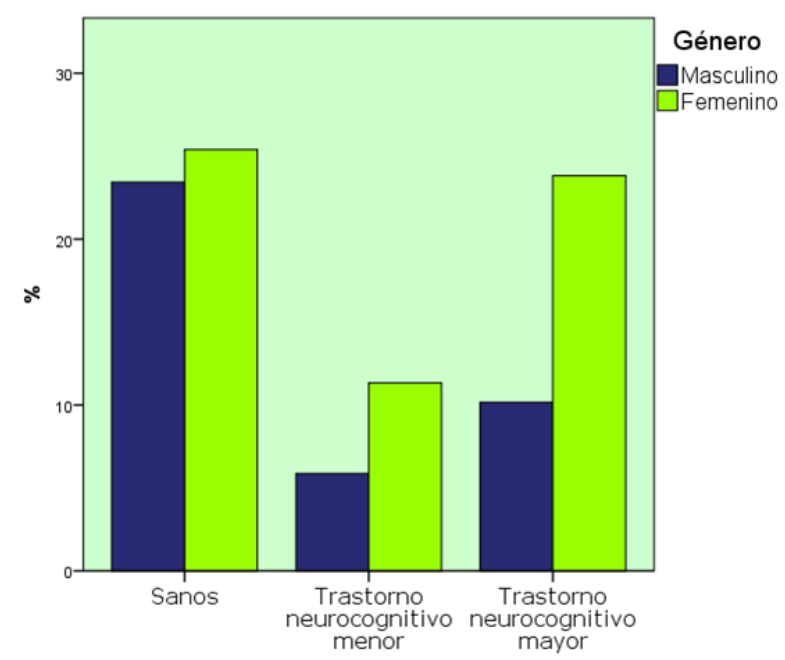

Figura $\mathrm{N}^{\circ}$ 1. Porcentaje de trastornos neurocongnitivos en función del sexo. 
Para analizar la asociación entre los años de escolaridad y la presencia de TNC, se agruparon los años de educación en intervalos de cinco años: los sujetos que habían completado entre 1 y 5 años de escolaridad presentaron un 63,5\% ( $n=137)$; de 6 a 10 años, un $34,95 \%(n=103)$ y con 11 y más años, un $50 \%$ de TNC, pero con un número sensiblemente menor de adultos mayores, $\mathrm{n}=16$. Teniendo en cuenta el tipo de TNC, las personas con mayor educación no cumplieron con los criterios diagnósticos para un TNC mayor, sino más bien con los del menor (fig. 2).

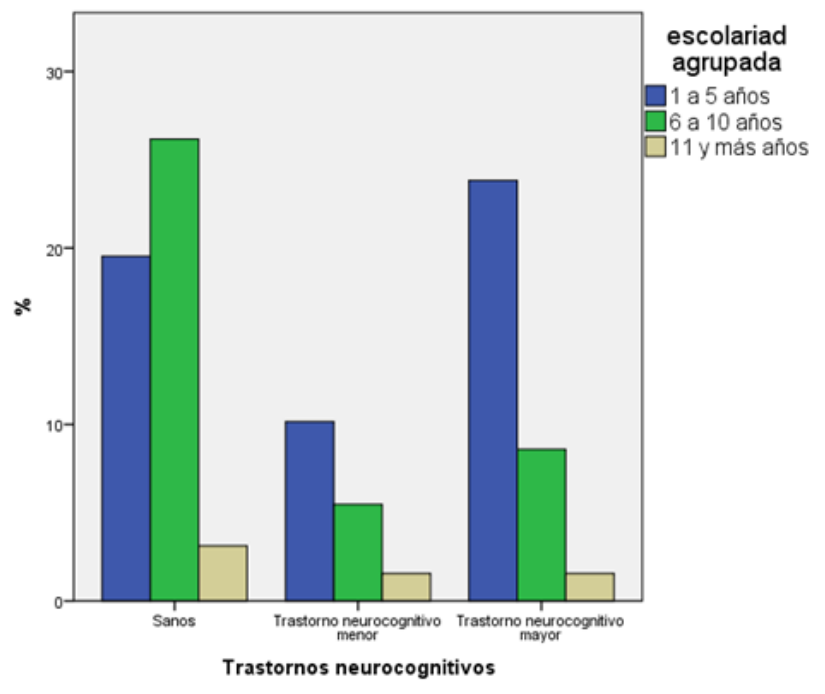

Figura $\mathbf{N}^{\circ}$ 2. Distribución de trastornos neurocongnitivos según escolaridad.

En cuanto a la edad, se dividió a los participantes en dos grupos: adultos mayores-jóvenes (60-79 años) y adultos mayores-mayores (80 y más años), acorde con la literatura en la materia ${ }^{(27,28)}$, que expresa una aumento de la prevalencia conforme avanza la edad. Se utilizó el estadístico Tau-c de Kendal para observar la asociación entre las variables, siendo ésta significativa, $\mathrm{T}(257)=.139$, p.00. Al analizar la presencia de trastornos neurocognitivos en función de los antecedentes clínicos de las personas, no se encontraron asociaciones significativas (Tabla 1 ).

\section{Tabla $\mathrm{N}^{\circ} 1$ : Asociación de variables clínicas con la presencia de trastornos neurocognitivos. Se tuvieron en cuenta tres categorías: Sin TNC, TNC menor y TNC mayor}

\begin{tabular}{ccc}
\hline & Chi $^{\mathbf{2}}$ & Sig. \\
\hline Hipertensión arterial & 1,62 & 0,445 \\
Enfermedad tiroidea & 0,926 & 0,629 \\
Parkinsonismo & 0,717 & 0,699 \\
Diabetes & 2,95 & 0,229 \\
Dislipemia & 4,879 & 0,087 \\
ACV & 1,95 & 0,377 \\
TEC & 0,143 & 0,931 \\
Enfermedad cardíaca & 4,715 & 0,095 \\
Consumo de alcohol & 0,805 & 0,669 \\
Tabaquismo & 3,232 & 0,199 \\
Antecedentes psiquiátricos & 12,73 & 0,002 \\
Antecedentes familiares de & 0,212 & 0,899 \\
\hline demencia &
\end{tabular}

Nota: $\mathrm{ACV}=$ accidente cerebro vascular; $\mathrm{TEC}=$ traumatismo encéfalo craneano

\section{DISCUSIÓN}

El presente trabajo tuvo como objetivo estimar la prevalencia de trastornos neurocognitivos en adultos mayores de 60 años pertenecientes al área Este de la provincia de Tucumán durante el año 2018 , siendo esta de $51,17 \%$. Los datos obtenidos superan ampliamente la estimación esperada en la República Argentina, que es del $12 \%{ }^{(19)}$. A partir de la interpretación de los resultados a la luz de las características sociodemográficas de la población, se observa que en general los sujetos mostraron un bajo nivel de educación formal, con presencia de analfabetismo. Aproximadamente el $20 \%$ de los sujetos aspirantes a participar de este estudio, nunca habían sido alfabetizados, por lo que fueron excluidos del estudio, lo que redujo el intervalo de confianza del estudio al $90 \%$, siendo menor que lo esperado, pero aceptable para este tipo de trabajos, sobre todo por ser realizado a partir de fuentes primarias.

Uno de los vectores para analizar la alta prevalencia estimada, tiene que ver con las características de la muestra utilizada en este estudio. El área Este se caracteriza por ser una zona rural, con pueblos aledaños a ingenios, donde históricamente la ocupación principal estuvo asociada a la producción azucarera ${ }^{(7,18)}$. En este rubro, es frecuente observar que la inserción al mundo laboral comenzaba a edades muy tempranas. Los sujetos que participaron de este trabajo y que tenían estudios primarios incompletos, hicieron referencia a que no habían podido terminar de estudiar por su inserción al mercado laboral a temprana edad y, que esto hacía incompatible su continuidad en los establecimientos escolares. Asimismo, en estos lugares las escuelas suelen quedar alejadas de los pequeños asentamientos aledaños a la industria azucarera, razón por la cual los participantes manifestaron haber tenido que abandonar sus estudios luego de la primaria, haciendo referencia a que, para poder continuar con los estudios secundarios, debían mudarse a zonas más populosas y no tenían los recursos para ello. La asociación entre la ruralidad y los TNC presenta resultados contradictorios. Una revisión sistemática reciente ${ }^{(7)}$ mostró que en América Latina son menos frecuentes los casos de TNC en población rural respecto de aquella de procedencia urbana. Por otro lado, otros estudios ${ }^{(29)}$ de prevalencia han encontrado una cantidad significativamente mayor de casos en áreas rurales sobre las urbanas. La hipótesis más fuerte a este respecto está en relación con las diferencias en los años de educación, apoyando la hipótesis de la reserva cognitiva ${ }^{(15)}$ que expresa que un mayor nivel educativo actuaría como un factor protector ante las enfermedades neurodegenerativas. Asimismo, otras posibles explicaciones se encuentran en la forma de tomar los datos. El estudio ReDeCar ${ }^{(9)}$ tomó sus datos a partir de consultas hospitalarias. Basados en estudios anteriores realizados en poblaciones rurales $^{(6,17,29)}$, creemos que la naturalización del deterioro cognitivo en la vejez en éstas áreas, así como la dificultad de acceso a efectores de salud especializados que atiendan este tipo de problemáticas son factores que reducen las consultas efectivas que realizan las personas mayores y sus familiares por quejas cognitivas. Este estudio abordó la problemática yendo al hogar de las personas mayores a tomar los datos, por lo que consideramos estima más fielmente la realidad del área Este de la provincia de Tucumán.

A propósito de lo anterior, una importante limitación en este estudio es que sus datos corresponden a un área circunscripta dentro de la provincia de Tucumán con características particulares y distintas del resto del territorio. De los participantes, la mayoría tenían estudios primarios completos (7 años) o incompletos, y no accedieron a una educación posterior. Por ello, se estima que la representatividad de los resultados en el área Este, no pueden generalizarse al resto de la provincia. Queda por delante la replicación del estudio en el resto del territorio provincial con el objetivo de generar datos certeros y fidedignos.

Excluyendo a los sujetos que no habían sido alfabetizados, aún había un $80 \%$ de la muestra que nunca cursó estudios de bachiller o secundarios. Al analizar estos datos con los resultados obtenidos en la pruebas cognitivas de screening de la ENP, se observó que a menor nivel educativo y a mayor edad(7), aumenta el riesgo para 
desarrollar trastornos neurocognitivos, mientras que un mayor nivel educativo ${ }^{(14-16)}$ actuaría como un factor protector para este tipo de patologías. No se puede establecer esta conclusión de manera definitiva, debido a que la muestra recabada en este trabajo con alto nivel educativo fue muy escasa. Sin embargo, estos resultados apoyan lo descrito por Zurique-Sánchez ${ }^{(7)}$, al decir que la evidencia científica sugiere la existencia de una correlación inversa entre los años de estudio formales y el padecimiento de un TNC, indicando que a menor escolaridad, mayor riesgo de demencia. Además, los autores relacionan no sólo la educación sino la ocupación laboral y explicaron que las actividades laborales predominantemente manuales se relacionarían con el inicio y la velocidad de progresión del declive cognitivo en la edad avanzada, tal como explican otros autores $^{(12)}$. En este estudio, como se ha mencionado anteriormente, existe un gran porcentaje de sujetos que han dedicado su vida como operarios en la industria azucarera. Estas consideraciones permiten explicar en parte la alta prevalencia de TNC en el área Este.

En cuanto a la distribución por género de trastornos neurocognitivos, el género femenino aparece como controversial a la hora de considerarse como factor de riesgo ${ }^{(7,13)}$. Si bien el presente estudio muestra mayor porcentaje de mujeres con trastornos neurocognitivos, la muestra total es en su mayoría de mujeres. Además, hay que tener en cuenta el bajo acceso a la educación y al trabajo de la población femenina estudiada y, si se considera que estos factores forman parte de la "reserva

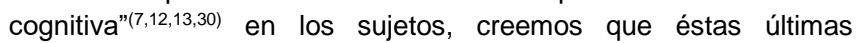
variables explican de manera más acertada las diferencias encontradas entre ambos sexos ${ }^{(13)}$.

En este estudio no se encontraron correlaciones significativas con comorbilidades, por lo que se disiente con algunos autores ${ }^{(7)}$ que establecen como factores de riesgo el tabaquismo, la obesidad y la diabetes mellitus. En general, la asociación con comorbilidades se asienta en estudios con poblaciones más numerosas que las aquí analizadas. Por ello, queda por delante aumentar la muestra y tener en cuenta las características de esta población por su ubicación y ocupación principal para contrastar con más fuerza estos supuestos.

Para concluir, se acuerda con lo planteado por los autores del Re.De.Car ${ }^{(9)}$, puesto que este trabajo demuestra la capacidad de desarrollar estudios epidemiológicos a gran escala en el sistema sanitario público, articulando la actividad de los diferentes centros de salud. Los resultados aquí encontrados suponen una señal de alerta sobre el sistema público de salud y compelen a iniciar programas de prevención secundaria y terciaria a la vez que se refuerzan y crean programas de prevención primaria para las generaciones que actualmente se encuentran envejeciendo.

\section{Agradecimientos:}

Este proyecto fue llevado a cabo en el marco de una beca individual titulada "Abraam Sonis", del Ministerio de Salud de la Nación, otorgada en el año 2018. La beca de la que esté artículo es la conclusión natural se titula "Estudio de prevalencia de trastornos neurocognitivos en adultos mayores del área programática este en la provincia de Tucumán durante el año 2018" y fue desarrollada durante el período 2018-2019.

\section{Limitaciones de responsabilidad:}

La responsabilidad de este trabajo es exclusivamente de los autores.

\section{Conflicto de interés:}

Ninguno

\section{Fuentes de apoyo:}

La presente investigación fue realizada con el apoyo financiero del Ministerio de Salud de la Nación, producto de la beca de investigación Abraam Sonis, llevada a cabo en el período 2018-2019.

\section{Originalidad:}

Este artículo es original y no ha sido enviado para su publicación a otro medio de difusión científica en forma completa ni parcialmente.

\section{Cesión de derechos:}

Los participantes de este trabajo ceden el derecho de autor a la Universidad Nacional de Córdoba para publicar en la RFCM y realizar las traducciones necesarias.
Contribucion de los autores:

Todos los autores han participado en la concepción del diseño, recolección de la información y elaboración del manuscrito, haciéndose públicamente responsables de su contenido y aprobando su versión final.

\section{BIBLIOGRAFÍA}

1. United Nations. World population prospects 2019: highlights. Department of Economic and Social Affairs, Population Division; 2019 Disponible

en: https://www.un.org/development/desa/publications/world-populationprospects-2019-highlights.html

2. United Nations. Department of Economic and Social Affairs. World. Population Ageing 2015: (ST/ESA/SER.A/390). Disponible

https://www.un.org/en/development/desa/population/publications/pdf lageing/WPA2015 Report.pdf

3. Organización Mundial de la Salud. Acción multisectorial para un envejecimiento sano basado en el ciclo de vida: proyecto de estrategia y plan de acción mundiales sobre el envejecimiento y la salud. In 69. ‥ Asamblea Mundial de la Salud, Ginebra, 22 Abr 2016 A69/17. Disponible http://apps.who.int/gb/ebwha/pdf files/WHA69/A69 17-sp.pdf

4. Organización Panamericana de la Salud. Organización Mundial de la Salud. Plan de acción sobre la salud de las personas mayores, incluido el envejecimiento activo y saludable: informe final. Report No.: CD57/INF/9. Washington: OPS. OMS. $57^{\circ}$ Consejo Directivo; 2019. Disponible en: https://www3.paho.org/hq/index.php?option=com docman\&view=do wnload\&alias=49692-cd57-inf-9-s-pda-personasmayores\&category slug=cd57-es\&/temid=270\&lang=es

5. American Psychiatric Association. Diagnostic and statistical manual of mental disorders (DSM-5®): American Psychiatric Pub.; 2013.

6. Petersen MS, Restorff M, Stórá $T$, Waldemar $G$, Joensen $S$. Trend in the Incidence and Prevalence of Dementia in the Faroe Islands. J Alzheimers Dis. 2019;71(3):969-978. doi: 10.3233/JAD190341.

7. Zurique Sánchez C, Cadena Sanabria MO, Zurique Sánchez M, Camacho López PA, Sanchez Sanabria M, Hernández Hernández S, Velásquez Vanegas K, Ustate Valera A. Prevalencia de demencia en adultos mayores de América Latina: revisión sistemática. Rev Esp Geriatr Gerontol. 2019; 54(6):346-355. doi: 10.1016/j.regg.2018.12.007.

8. Aranda $M$, Calabria A. Impacto económico-social de la enfermedad de Alzheimer. Neurología Argentina. 2019; 11(1):19-26. doi:: 10.1016/j.neuarg.2018.11.001

9. Ministerio de Salud de la Nación. Presidencia de la Nacion. Primer registro centralizado de patologías cognitivas en Argentina ReDeCAR: Resultados del estudio piloto. Sistema de vigilancia epidemiológica en Salud mental y adicciones. Buenos Aires: Ministerio de Salud de la Nación; 2011; 5.

10. Block CK, Johnson-Greene D, Pliskin N, Boake C. Discriminating cognitive screening and cognitive testing from neuropsychological assessment: implications for professional practice. Clin Neuropsychol. 2017 Apr;31(3):487-500. doi: 10.1080/13854046.2016.1267803.

11. Luna-Lario P, Azcárate-Jiménez L, Seijas-Gómez R, TirapuUstárroz J. Propuesta de una batería neuropsicológica de evaluación cognitiva para detectar y discriminar deterioro cognitivo leve y demencias. Rev Neurol. 16;60(12):553-61.

12. Sörman DE, Hansson $P$, Pritschke I, Ljungberg JK. Complexity of Primary Lifetime Occupation and Cognitive 
Processing. Front Psychol. 2019 Aug 21; 10:1861. doi: 10.3389/fpsyg.2019.01861.

13. Ardila A, Ostrosky F. Guía para el diagnóstico neuropsicológico. Florida; American Board of Professional Neuropsychology. 2012. Disponible en: http://ineuro.cucba.udg.mx/libros/bv guia para el diagnostico neur opsicologico.pdf.

14. Chen Y, Lv C, Li X, Zhang J, Chen K, Liu Z, Li H, Fan J, Qin $T$, Luo $L$, Zhang $Z$. The positive impacts of early-life education on cognition, leisure activity, and brain structure in healthy aging. Aging (Albany NY). 2019 Jul 17;11(14):4923-4942. doi: 10.18632/aging.102088.

15. Lu H, Wang XD, Shi Z, Yue W, Zhang Y, Liu S, Liu S, Zhao L, Xiang L, Zhang Y, Guan Y, Su W, Li Z, Wang J, Wisniewski T, Ji Y. Comparative analysis of cognitive impairment prevalence and its etiological subtypes in a rural area of northern China between 2010 and 2015. Sci Rep. 2019 Jan 29;9(1):851. doi: 10.1038/s41598-01837286-z.

16. Mukadam N, Sommerlad A, Huntley J, Livingston G. Population attributable fractions for risk factors for dementia in lowincome and middle-income countries: an analysis using crosssectional survey data. The Lancet Global Health. 2019;7(5):e596e603. doi: 10.1016/S2214-109X(19)30074-9.

17. solaki M, Gkioka M, Verykouki E, Galoutzi N, Kavalou E, Pattakou-Parasyri V. Prevalence of Dementia, Depression, and Mild Cognitive Impairment in a Rural Area of the Island of Crete, Greece. Am J Alzheimers Dis Other Demen. 2017 Aug;32(5):252-264. doi: 10.1177/1533317517698789.

18. Deng J, Cao C, Jiang Y, Peng B, Wang T, Yan K, Lian J, Wang Z. Prevalence and effect factors of dementia among the community elderly in Chongqing, China. Psychogeriatrics. 2018 Sep;18(5):412-420. doi: 10.1111/psyg. 12343.

19. Ministerio de Salud de la Nación. Estado de conocimiento y agenda de prioridades para la toma de decisiones en Enfermedades Neurológicas. Enfermedad Cerebro vascular en Argentina. Buenos Aires; 2008.2 Disponible en: https://healthresearchweb.org/?action=download\&file=2008-Ecv.pdf

20. American Psychological Association. Ethical principles of psychologists and code of conduct (2002, amended effective June 1, 2010, and January 1, 2017); 2017. Disponible en: https://www.apa.org/ethics/code/ethics-code-2017.pdf.

21. Manzini JL. Declaración de Helsinki: Principios éticos para la investigación médica sobre sujetos humanos. Acta bioethica. 2000; 6(2):321-334. doi: 10.4067/S1726-569X200000020001.

22. Butman J, Arizaga RL, Harris $P$, Drake $M$, Baumann $D$, dePascale A, Allegri RF, Mangone CA, Ollari JA. El "Mini-Mental State Examination" en español. Normas para Buenos Aires. Rev Neurol Arg. 2001; 26(1):11-15.

23. Cacho-Guetiérrez LJ, García-García R, Arcaya-Navarro J, Gay-Puente FJ, Guerrero AL, Gómez-Sánchez JC, Vicente-Vilardón JL. El test del reloj en ancianos sanos. Rev Neur. 1996; 24(136):1525-1528. doi: 10.33588/rn.24136.96404.

24. Labos E, Trojanowski S, del Rio M, Zabala K, Renato A. Perfiles de fluencia verbal en Argentina. Caracterización y normas en tiempo extendido. Neurología Argentina. 2013; 5(2):78-86. doi: 10.1016/j.neuarg.2013.04.005

25. Fernández AL, Marino J, Alderete A. Estandarización y validez conceptual del Test del Trazo en una muestra de adultos argentinos. Revista neurológica argentina. 2002; 27(2):83-88.

26. IBM Corp. [IBM SPSS Statistics for Windows, versión 23.0 [software de cómputo]]. Armonk.

27. Kodesh A. Prevalence and comorbidities of dementia in Israel: A nationally representative cohort study. International
Psychogeriatrics. Cambridge University Press. 2019; 31(7):1059-63. https://doi.org/10.1017/S1041610218001461.

28. Ponjoan A, Garre-Olmo J, Blanch J, Fages E, AlvesCabratosa L, Martí-Lluch R, Comas-Cufí M, Parramon D, Garcia-Gil $M$, Ramos R. Epidemiology of dementia: prevalence and incidence estimates using validated electronic health records from primary care. Clin Epidemiol. 2019 Mar 4; 11:217-228. doi: 10.2147/CLEP.S186590.

29. Jia J, Wang F, Wei C, Zhou A, Jia X, Li F, Tang M, Chu L, Zhou $Y$, Zhou $C$, Cui $Y$, Wang $Q$, Wang $W$, Yin $P$, Hu $N$, Zuo $X$, Song $H$, Qin W, Wu L, Li D, Jia L, Song J, Han Y, Xing Y, Yang P, Li Y, Qiao $Y$, Tang $Y, L v J$, Dong $X$. The prevalence of dementia in urban and rural areas of China. Alzheimers Dement. 2014 Jan;10(1):1-9. doi: 10.1016/j.jalz.2013.01.012.

30. Stern Y. Cognitive reserve. Neuropsychologia. 2009 Aug;47(10):2015-28. doi: 10.1016/j.neuropsychologia.2009.03.004. 